\title{
Massive infarction and cavernous sinus thrombosis: an uncommon complication of tooth extraction
}

\author{
Umamaheswara Reddy V. ${ }^{1}$, Amit Agrawal ${ }^{2}$, Kishor V. Hegde ${ }^{3}$, \\ Srikanth V. ${ }^{4}$, Sahith Reddy K. ${ }^{4}$ \\ Narayana Medical College Hospital, Chinthareddypalem, Nellore, Andhra Pradesh, India \\ ${ }^{1}$ Assistant Professor of Radiology, Department of Radiology \\ ${ }^{2}$ Professor of Neurosurgery, Department of Neurosurgery \\ ${ }^{3}$ Professor of Radiology, Department of Radiology \\ ${ }^{4}$ Resident of Radiology, Department of Radiology
}

\begin{abstract}
Cavernous sinus thrombosis (CAST) is a rare and potentially fatal complication following tooth extraction. In present case of a 55 year old male known case of diabetes mellitus underwent tooth extraction. After 5 days, he noticed swelling around the cheek, high grade fever, and frontal headaches. Diagnosis of alveolar abscess after inspection was made, for which incision and drainage was done. Next morning, he noticed that the cheek swelling progressed to left eye and there was CT brain was performed which showed hyperdense areas in cavernous sinus on left side and left sylvian fissure with hemorrhagic venous infarct in left temporal and frontal lobes. More caudal sections revealed mucosal thickness in left maxillary and ethmoidal sinuses, edema over cheek, preseptal orbital swelling, retro-orbital fat stranding and axial proptosis. A diagnosis of rhino-orbital infection from dental source with cavernous sinus extension causing left temporo-frontal hemorrhagic venous infarction was made Emergency surgery for decompression was performed but the patient did not responded to the treatment and succumbed to the infection. This case is a reminder that in patients with uncontrolled diabetes, undergoing dental procedures should be carefully dealt with appropriate antibiotic cover. Early signs like unilateral facial edema, orbital chemosis, edema, and proptosis should raise high index suspicion of cavernous sinus thrombosis. Key words: Cavernous sinus thrombosis, venous infarction, Diabetes Mellitus, Dental infection.
\end{abstract}

\section{Introduction}

Cavernous sinus thrombosis (CAST) is a rare and potentially fatal complication following tooth extraction. (1-4)
In present case we report a rare complication of tooth extraction where patient developed cavernous sinus thrombosis with resultant fatal outcome. 


\section{Case report}

A 55 year old male known case of diabetes mellitus went to the dental OPD in rural hospital with left sided tooth ache. He was diagnosed to have cavity in the left second upper premolar: 25 which was extracted. $\mathrm{He}$ was prescribed oral analgesics and antibiotics (Ampicillin -500 mg twice daily). After 5 days, he reported to the dentist for swelling around the cheek, high grade fever, frontal headaches. Diagnosis of alveolar abscess after inspection was made, for which incision and drainage was done. Patient was referred to diabetologist for his high blood sugar of $369 \mathrm{mg} / \mathrm{dl}$. Patient postponed the meet with doctor as he got some symptomatic relief with the analgesics. Next morning, he noticed that the cheek swelling progressed to left eye and there was purulent discharge from left eye. While patients' relatives were making necessary arrangements to shift him to tertiary care center, he developed right side hemiparesis. During the transportation, condition of the patient rapidly deteriorated and patient was brought to our hospital with poor GCS - 5. On examination, he had swelling of left cheek, eyelid necrosis and his neurological examination revealed decreased tone on right side. Vital parameters at the time of arrival were - Pulse -114bpm, Blood pressure-70/30 mmHg, RBS- 409 gm/dl, RR-30 cpm, SpO2$85 \%$. Tone and reflexes were decreased on the right side. Routine investigations showed ketonuria, WBC-20000/mm3 (Neutrophil$75 \%$, Lymphocytes-23\%, Monocytes-2\%), Plts-1.5 lacs. CT brain was performed which showed hyperdense areas in cavernous sinus on left side and left sylvian fissure with hemorrhagic venous infarct in left temporal and frontal lobes. More caudal sections revealed mucosal thickness in left maxillary and ethmoidal sinuses, edema over cheek, preseptal orbital swelling, retro-orbital fat stranding and axial proptosis. A diagnosis of rhino-orbital infection from dental source with cavernous sinus extension causing left temporo-frontal hemorrhagic venous infarction was made. In view of extensive mass effect and compression on ipsilateral cerebral peduncle. Emergency surgery for decompression was performed. However there was no improvement of GCS after the surgery and patient succumbed to death.

\section{Discussion}

Intracranial infection from odontogenic source resulting in CAST is rare and contributes only upto $10 \%$ cases of CAST. (1, 3) Spread of extracranial infection to cavernous sinus can occur from paranasal sinuses, orbits, teeth, periodontal tissues, skin over face and cheek is facilitated by network of valve less venous plexus in which blood flow is pressure gradient dependent. (5) Dental pathogens and septic emboli can spread into cavernous sinus through pterygoid venous plexus. (3) Numerous bacterial (aerobic and an-aerobic) and fungal pathogens can cause cavernous sinus thrombosis. Staphylococcus is most common organism causing cavernous sinus thrombosis. $(4,6)$ 


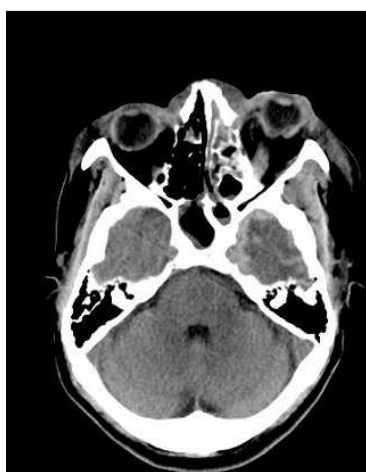

A

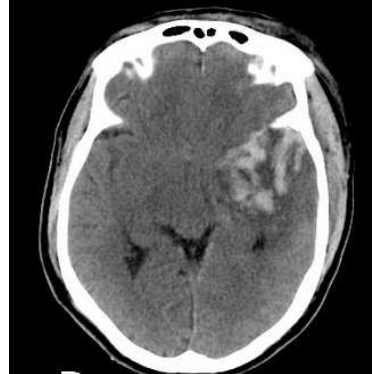

D

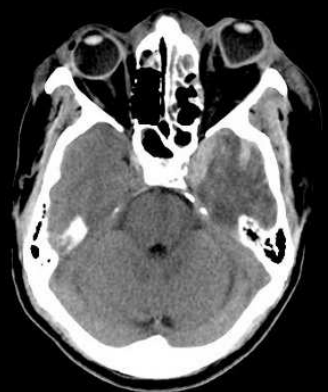

$\mathbf{B}$

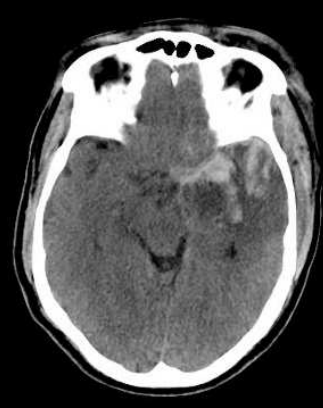

C

Figure 1 - Axial CT images (A, B) showing hyperdense material within the left cavernous sinus, cavernous sinus is showing convex borders and axial orbital proptosis. Axial CT Images (C, D) showing blood within the sylvian fissure.

Large variegated intraparenchymal hemorrhage is seen in left temporo-frontal lobes causing midline shift (E, F)

Occurrence of CAST multiplies by many folds in immunocompromised patients, as normal flora in otherwise healthy individuals become pathogenic due to ineffective defense mechanisms. (7) Even minor infections in head and neck region in these patients should be judiciously treated to prevent this life threatening complications. (5) High index suspicion is required to have a diagnosis of CAST: unilateral facial swelling, chemosis and acute onset of proptosis are warning signs which should raise the suspicion. (5) Contrast CT scan or Contrast MRI is mainstay in diagnosing this condition. (4) Filling defects within the cavernous sinus, Convex bulge of the cavernous sinus, dilated ophthalmic vein are typical imaging signs. Imaging should also be targeted to look for later consequences of CAST like meningitis, empyemas, infarctions, aneurysms of cavernous sinus, fistulas etc. (4, 5) Once diagnosis of cavernous sinus thrombosis is established, broad spectrum antibiotics should be empirically started as any delay in due process can result in devastating complications. However, antibiotics should be tailored later according to the culture reports. (3) Role of anti-coagulants in treatment is controversial. (8) Mortality and morbidity after effective treatment is still high. 

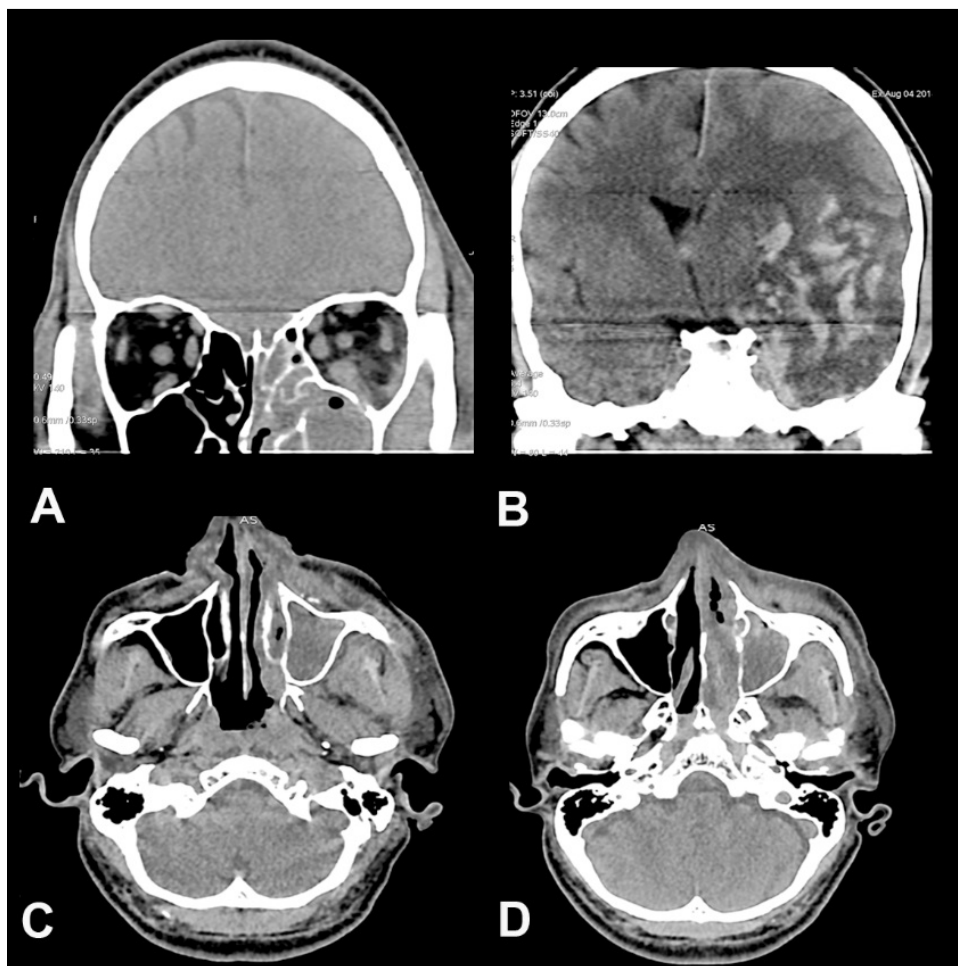

B

Figure 2 - Coronal reconstructed CT scan images (A, C, D) showing iso-hyperdense material within the left maxillary sinus and left retro orbital inflammation suggestive of cellulitis. Cranio-caudal extensions of intraparenchymal hemorrhage and midline shift are better appreciated on image (B)

This case is a reminder that in patients with uncontrolled diabetes, undergoing dental procedures should be carefully dealt with appropriate antibiotic cover. Early signs like unilateral facial edema, orbital chemosis, edema, and proptosis should raise high index suspicion of cavernous sinus thrombosis. Time is most important while dealing these cases as the initial manifestations may be subtle but there may be rapid clinical deterioration which can occur within hours of onset.

\section{Correspondence}

Dr. Amit Agrawal

Professor of Neurosurgery
Department of Neurosurgery

Narayana Medical College Hospital

Chinthareddypalem

Nellore-524003

Andhra Pradesh (India)

Email-dramitagrawal@gmail.com

dramit_in@yahoo.com

Mobile- +91-8096410032

\section{References}

1. Ogundiya DA, Keith DA, Mirowski J. Cavernous sinus thrombosis and blindness as complications of an odontogenic infection: report of a case and review of literature. Journal of oral and maxillofacial surgery: official journal of the American Association of Oral and Maxillofacial Surgeons 1989;47:1317-1321. 
DOI: 10.2478/romneu-2014-0069

2. Okamoto H, Ogata A, Kosugi M, Takashima H, Sakata S, Matsushima T. Cavernous sinus thrombophlebitis related to dental infection--two case reports. Neurologia medico-chirurgica 2012;52:757-760.

3. Verma R, Junewar V, Singh RK, Ram H, Pal US. Bilateral cavernous sinus thrombosis and facial palsy as complications of dental abscess. National journal of maxillofacial surgery 2013;4:252.

4. Ebright JR, Pace MT, Niazi AF. Septic thrombosis of the cavernous sinuses. Archives of Internal medicine 2001;161:2671-2676.

5. Jones RG, Arnold B. Sudden onset proptosis secondary to cavernous sinus thrombosis from underlying mandibular dental infection. BMJ case reports 2009;2009:bcr0320091671.

6. Kamouchi M, Wakugawa Y, Okada Y, et al. Venous infarction secondary to septic cavernous sinus thrombosis. Internal medicine (Tokyo, Japan) 2005;45:25-27.

7. Fehrenbach MJ, Herring SW. Spread of dental infection. Practical Hygiene 1997;6:13-19.

8. Bhatia K, Jones N. Septic cavernous sinus thrombosis secondary to sinusitis: are anticoagulants indicated? A review of the literature. The Journal of Laryngology \& Otology 2002;116:667-676. 